Nagoya Math. J., 222(1) (2016), 74-99

DOI $10.1017 / \mathrm{nmj} .2016 .11$

\title{
BERNSTEIN-SATO POLYNOMIALS AND TEST MODULES IN POSITIVE CHARACTERISTIC
}

\author{
MANUEL BLICKLE AND AXEL STÄBLER
}

\begin{abstract}
In analogy with the complex analytic case, Mustaţă constructed (a family of) Bernstein-Sato polynomials for the structure sheaf $\mathcal{O}_{X}$ and a hypersurface $(f=0)$ in $X$, where $X$ is a regular variety over an $F$-finite field of positive characteristic (see Mustaţă, Bernstein-Sato polynomials in positive characteristic, J. Algebra 321(1) (2009), 128-151). He shows that the suitably interpreted zeros of his Bernstein-Sato polynomials correspond to the $F$-jumping numbers of the test ideal filtration $\tau\left(X, f^{t}\right)$. In the present paper we generalize Mustaţă's construction replacing $\mathcal{O}_{X}$ by an arbitrary $F$-regular Cartier module $M$ on $X$ and show an analogous correspondence of the zeros of our Bernstein-Sato polynomials with the jumping numbers of the associated filtration of test modules $\tau\left(M, f^{t}\right)$ provided that $f$ is a nonzero divisor on $M$.
\end{abstract}

\section{Introduction}

To keep notation simple in this introduction let $X=\operatorname{Spec} k\left[x_{1}, \ldots, x_{n}\right]$ be the affine $n$-space over an algebraically closed field $k$. Denote the polynomial ring by $R=k\left[x_{1}, \ldots, x_{n}\right]$ and fix an equation $f \in R$ defining a hypersurface in $X$. We denote by $\gamma:$ Spec $R \rightarrow$ Spec $R[t]$ the graph embedding of $f$ given by sending $t$ to $f$.

If $k=\mathbb{C}$ one has the Bernstein-Sato Polynomial of $f$ which is an important measure of the singularities of the hypersurface defined by $f=0$. It is defined to be the nonzero monic polynomial of minimal degree among those $b(s) \in k[s]$ such that

$$
b(s) f^{s}=P f^{s+1}
$$

for some differential operator $P \in \mathcal{D}_{R}[s]=k\left[x_{1}, \ldots, x_{n}, \partial_{x_{1}}, \ldots, \partial_{x_{n}}\right][s]$.

Kashiwara and Malgrange interpret in [16] and [21] the Bernstein-Sato polynomial as the minimal polynomial of the action of the Euler operator

Received February 12, 2014. Accepted April 19, 2015.

2010 Mathematics subject classification. Primary 13A35; Secondary 14F10.

Both authors were supported by SFB/Transregio 45 Bonn-Essen-Mainz financed by Deutsche Forschungsgemeinschaft.

(C) 2016 by The Editorial Board of the Nagoya Mathematical Journal 
$\frac{\partial}{\partial t} t$ on a graded piece of the $V$-filtration of the $\mathcal{D}_{R}$-module pushforward $\gamma_{+} R$ along the graph embedding. In fact, a key point in the work of Kashiwara and Malgrange is the construction of said $V$-filtration in a much more general context, namely for regular holonomic $\mathcal{D}_{R}$-modules, which they achieve by their theory of $b$-functions, which generalizes the BernsteinSato polynomial for a hypersurface equation recalled above. By work of Budur and Saito [12] from the $V$-filtration on the $\mathcal{D}_{R[t]}$-module $\gamma_{+} R$, one can reconstruct the filtration of multiplier ideals $\mathcal{J}\left(R, f^{t}\right) \subseteq R$ for $0<t \leqslant 1$. This shows, in particular, that the jumping numbers of the multiplier ideal filtration between 0 and 1 are zeros of the Bernstein-Sato polynomial.

A consequence of the existence of the Bernstein-Sato Polynomial is that the $\mathcal{D}_{R}$-module $R_{f}$ is generated by $1 / f$ if (and only if) the reduced Bernstein-Sato Polynomial $(x+1)^{-1} b(s)$ does not have negative integral roots [27]. However, if $k$ is a field of positive characteristic $p>0$, then it is shown in [1] that the $\mathcal{D}_{R}$-module $R_{f}$ is always generated by $1 / f$. Hence, there cannot be a theory of Bernstein-Sato polynomials in positive characteristic with the same defining property. This observation is just one example for the fact that $\mathcal{D}$-module theory in positive characteristic is quite different from the complex case.

However, by taking the interpretation of the Bernstein-Sato polynomial as the minimal polynomial of an action of the Euler operator (due to Kashiwara and Malgrange) as his point of departure, Mustaţă defines in [23] a family of Bernstein-Sato polynomials for a hypersurface $f=0$ over a field of positive characteristic. Contrary to the complex analytic case it is not enough to consider the action of the Euler operator alone; instead one has to also consider all higher divided power Euler operators $\vartheta_{i}=\partial_{t}^{\left[p^{i}\right]} t^{p^{i}}$ at once. $^{1}$

More precisely, for $e \geqslant 1$ let $M_{f}^{e}$ be the $\mathcal{D}_{R}^{e}\left[\vartheta_{1}, \ldots, \vartheta_{p^{e-1}}\right]$-module generated by the image of $\gamma_{*} R$ in $\gamma_{+} R$, where $\mathcal{D}_{R}^{e}$ is the subring consisting of those differential operators which are linear over $R^{p^{e}}$. The Euler operators $\vartheta_{i}$ act on the quotient $M_{f}^{e} / t M_{f}^{e}$ for $1 \leqslant i \leqslant e-1$ with eigenvalues in $\mathbb{F}_{p}$. The eth Bernstein-Sato polynomial as introduced by Mustaţă encodes the common eigenvalues of these operators. Furthermore, Mustaţă proved that the information of these eigenvalues (suitably lifted to $\mathbb{Q}$ ) is equivalent to

\footnotetext{
${ }^{1}$ Note that the order is reversed here. That is, one usually considers $t^{p^{i}} \partial_{t}^{\left[p^{i}\right]}$. We will be able to use this standard convention once we switch to right modules.
} 
the data of the $F$-jumping numbers of the test ideal filtration $\tau\left(R, f^{t}\right)$ of $f$ in the range $(0,1]$. As the test ideal can be viewed as a positive characteristic analog of the multiplier ideal this statement is a characteristic $p$ version of the result of Budur and Saito that the jumping numbers of the multiplier ideal are zeroes of the classical Bernstein-Sato polynomial as alluded to above.

Work of Axel Stäbler in [25] suggests that in positive characteristic the test module filtration itself is a suitable analog of the $V$-filtration: For one thing there is a certain axiomatic characterization of the test module filtration similar to that of the $V$-filtration but also different in the sense that the action of the differential operators is replaced by a (right) action of the Frobenius. Furthermore, a certain associated graded piece of the test module filtration corresponds, via an analog of the RiemannHilbert correspondence, to a functor on perverse constructible sheaves of $\mathbb{F}_{p}$-vector spaces that has several of the desirable properties of nearby cycles in the $\ell \neq p$-case. This relationship between nearby cycles and $\mathcal{D}$-modules in characteristic 0 was the motivation behind the construction of the $V$ filtration for holonomic $\mathcal{D}$-modules as a way to realize the nearby cycles functor for constructible $\mathbb{C}$-sheaves on the $\mathcal{D}$-module side.

What we achieve in the present paper is to also generalize Mustaţă's theory of Bernstein-Sato polynomials to this more general context where the test module filtration is defined and well behaved as in [25]. In order to state our results let us recall some background on Cartier modules and their test modules from [6].

Let us from now on assume that $R$ is an $F$-finite Noetherian ring of positive characteristic $p$. A Cartier module $M$ (over $R$ ) is an $R$-module together with an $R$-linear map $\kappa: F_{*} M \rightarrow M$, where $F: R \rightarrow R$ is the absolute Frobenius given by $x \mapsto x^{p}$. A Cartier submodule of $M$ is an $R$-submodule $N$ such that $\kappa(N) \subseteq N$. We say that $M$ is $F$-pure if $\kappa$ is surjective. We call $M F$-regular if $M$ is $F$-pure and if for any Cartier submodule $N$ of $M$ which after localizing at every generic point of $\operatorname{Supp} M$ agrees with $M$ we have $N=M$.

Let $M$ be an $F$-regular Cartier module which as an $R$-module is finitely generated. Let $f$ be a nonzero divisor on $M$. Then the test module with respect to the ideal $(f) \subseteq R$ and $t \in \mathbb{R}_{\geqslant 0}$ is

$$
\tau\left(M, f^{t}\right)=\sum_{e \geqslant 1} \kappa^{e} f^{\left\lceil t p^{e}\right\rceil} f \underline{M_{\mathcal{C}}},
$$


where $\mathcal{C}$ is the algebra generated for $n \geqslant 1$ by the $\kappa^{n} f^{\left\lceil t p^{n}\right\rceil}$ and $M_{\mathcal{C}}=\left(\mathcal{C}_{+}\right)^{h} M$ for all $h \gg 0$ is the stable image (cf. [6, Proposition 2.13]). It follows from [6, Theorem 3.11], [25, Lemma 3.1] that the definition we give here is in fact equivalent to the definition of test modules in [6]. Moreover, we simplify the description of the test module in Section 4.

Test modules form a decreasing filtration of $R$-submodules of $M$, that is, $\tau\left(M, f^{s}\right) \subseteq \tau\left(M, f^{t}\right)$ for $s \geqslant t$. This filtration is right continuous, that is, for $\varepsilon \ll 1$ one has $\tau\left(M, f^{t}\right)=\tau\left(M, f^{t+\varepsilon}\right)$. An element $t \in \mathbb{Q}$ such that for all $\varepsilon>0$ one has $\tau\left(M, f^{t}\right) \neq \tau\left(M, f^{t-\varepsilon}\right)$ is called an $F$-jumping number (of the test module filtration along $f$ ). Test module filtrations satisfy the socalled Briançon-Skoda theorem, namely for any $t \geqslant 1$ one has $\tau\left(M, f^{t}\right)=$ $f \tau\left(M, f^{t-1}\right)$. In particular, it suffices to control the $F$-jumping numbers in the range $(0,1]$. Moreover, if $R$ is essentially of finite type over an $F$ finite field then the set of $F$-jumping numbers in $(0,1]$ is finite [6, Corollary $4.19]$ and all $F$-jumping numbers are rational (the rationality is a formal consequence of the finiteness similar to the argument in [9, Theorem 3.1]).

Similar to Mustaţă's approach in [23] we use the graph embedding along the fixed hypersurface $f=0$ to define a family of Bernstein-Sato polynomials $b_{f, M}^{e}(s) \in \mathbb{Q}[s]$. This will be done by exploiting a system of right $\mathcal{D}_{R}^{e}$-modules which arises from the Cartier module structure of $M$. This is explained in the following sections. Our main result can now be stated as follows:

THEOREM 5.4. Let $R$ be regular essentially of finite type over an $F$ finite field. Let $(M, \kappa)$ be an F-regular Cartier module and $f \in R$ a nonzero divisor on $M$. The roots of the Bernstein-Sato polynomials $b_{M, f}^{e}(s)$ are given for e sufficiently large by $\frac{\left[\lambda p^{e}\right\rceil-1}{p^{e}}$, where $\lambda$ varies over the $F$-jumping numbers of the test module filtration $\tau\left(M, f^{t}\right)$ for $t \in(0,1]$.

The crucial point is that the a priori infinite collection of BernsteinSato polynomials $b_{M, f}^{e}(s)$ for $e \geqslant 0$ is completely determined by a finite collection of rational numbers, namely the jumping numbers of the test module filtration attached to $(M, f)$.

In conclusion we would like to draw the reader's attention to the recent work of Stadnik [26] who also addresses the problem of extending Mustaţă's Bernstein-Sato polynomials to a more general context. Stadnik, however, works in the context of Emerton and Kisin's category of unit $R[F]$-modules [14]. In order to prove his existence result for $b$-functions he essentially has to reconstruct a theory of test ideals in this context, which he coins 
list-test-ideals in [26]. It was one motivation of the authors of the present paper to point out that, by working in the essentially equivalent theory of Cartier modules (more precisely Cartier crystals, see [7, Section 5.2]) one can rely on the already existing theory of test modules. By further replacing left $\mathcal{D}_{R}$-modules by right $\mathcal{D}_{R}$-modules there is a natural construction of the Bernstein-Sato polynomials with the desired link to the $F$-jumping numbers of the test module filtration. We show in Section 6 that Stadnik's $b$-functions are precisely the limit over our Bernstein-Sato polynomials $b_{M, f}^{e}(s)$.

\section{$\S 1 . \mathcal{D}$-modules in positive characteristic}

Throughout this article we assume all rings to contain a field of prime characteristic $p>0$. The absolute Frobenius homomorphism given by sending $r \mapsto r^{p}$ is denoted by $F: R \rightarrow F_{*} R$. For an $R$-module $M$ we denote by $F_{*}^{e} M$ the $R$-module whose underlying abelian group is $M$ but with multiplication given by $r \cdot m=r^{p^{e}} m$. The ring $R$ is called $F$-finite if $F_{*} R$ is a finite $R$-module; in other words, the Frobenius morphism on $\operatorname{Spec} R$ is a finite map.

Given a ring $R$ we denote by $\mathcal{D}_{R}$ the ring of (absolute) $\mathbb{Z}$-linear differential operators in the sense of Grothendieck [15]. Given a polynomial ring $R[t]$, we write $\partial_{t}^{[m]}: R[t] \rightarrow R[t]$ for the $R$-linear differential operator which sends $t^{n}$ to $\left(\begin{array}{c}n \\ m\end{array}\right) t^{n-m}$ with the usual convention that $\left(\begin{array}{c}n \\ m\end{array}\right)=0$ for $m>n$. We introduce the notation

$$
\theta_{m}=t^{m} \partial_{t}^{[m]} \quad \vartheta_{m}=\partial_{t}^{[m]} t^{m}
$$

for the $R$-linear operators which are given by sending $t^{n} \mapsto\left(\begin{array}{c}n \\ m\end{array}\right) t^{n}$ and $t^{n} \mapsto$ $\left(\begin{array}{c}n+m \\ m\end{array}\right) t^{n}$, respectively. The operator $\theta_{m}$ is called the (divided power) Euler operator of order $m$.

As $R$ is a ring of prime characteristic $p>0$ one has the $p$-filtration of its ring of differential operators, see [13]. The ring of (absolute) differential operators $\mathcal{D}_{R}$ is the direct limit of rings

$$
\mathcal{D}_{R}^{e} \cong \operatorname{End}_{R}\left(F_{*}^{e} R\right)
$$

called the differential operators of level $e$. Indeed, the inclusion from $\mathcal{D}_{R}^{e} \rightarrow$ $\mathcal{D}_{R}^{e+1}$ is the composition of the natural map $\operatorname{End}_{R}\left(F_{*}^{e} R\right) \rightarrow F_{*} \operatorname{End}_{R}\left(F_{*}^{e} R\right)$ followed by $F_{*} \operatorname{End}_{R}\left(F_{*}^{e} R\right) \rightarrow \operatorname{End}_{R}\left(F_{*}^{e+1} R\right)$. The direct limit over these maps yields $\mathcal{D}_{R}$.

If one uses $R^{p} \subseteq R$ instead of $R \rightarrow F_{*} R$ then one obtains the more familiar but equivalent description $\mathcal{D}_{R}^{e}=\operatorname{End}_{R^{p^{e}}}(R)$ and the union over these is $\mathcal{D}_{R}$. 
Also note that if $R[t]$ is a polynomial ring over $R$ then we have an inclusion $\mathcal{D}_{R} \rightarrow \mathcal{D}_{R[t]}$. Indeed, $F_{*} R[t]=\bigoplus_{i=0}^{p^{e}-1} F_{*} R t^{i}$ and given $P \in \mathcal{D}_{R}^{e}$ we have an extension $P^{\prime}$ by sending $b t^{i}$ to $P(b) t^{i}$ for $i \geqslant 0$.

We denote by Mod- $\mathcal{D}_{R}^{e}$ the category of right $\mathcal{D}_{R}^{e}$-modules and by Mod- $R$ the category of right $R$-modules.

We recall a theorem of Lucas $[19, \S \mathrm{XXI}]$ which states that given natural numbers $n, m$ with $p$-adic expansions $n=\sum_{i=0}^{s} a_{i} p^{i}$ and $m=\sum_{i=0}^{s} b_{i} p^{i}$ with $a_{i}, b_{i} \in\{0, \ldots, p-1\}$ one has

$$
\left(\begin{array}{l}
n \\
m
\end{array}\right)=\prod_{i=0}^{s}\left(\begin{array}{l}
a_{i} \\
b_{i}
\end{array}\right) \quad \bmod p
$$

In particular, it is a crucial ingredient in some proofs of the following relations among the differential operators in positive characteristic which we recall for the convenience of the reader.

LEMMA 1.1. Let $R$ be a regular and $F$-finite ring and $R[t]$ the polynomial ring over $R$ in one variable. Then the following hold:

(a) $\left[\partial_{t}^{\left[p^{i}\right]}, t^{p^{i}}\right]=1$ which just means $\vartheta_{i}=1+\theta_{i}$.

(b) $\frac{(s r) !}{(s !)^{r}} \partial_{t}^{[s r]}=\left(\partial_{t}^{[s]}\right)^{r}$.

(c) $\prod_{j=1}^{r}\left(\theta_{p^{e}}+j\right)=\left(\partial_{t}^{\left[p^{e}\right]}\right)^{r}\left(t^{p^{e}}\right)^{r}$.

(d) $\left[t, \theta_{p^{i}}\right]=-\theta_{p^{i}-1} t-t$ for all $i$.

(e) $\left[\theta_{i}, \theta_{j}\right]=0$ for all $i, j$.

(f) $\left[t, \theta_{m}\right]\left(t^{n}\right)=-\left(\begin{array}{c}n \\ m-1\end{array}\right) t^{n+1}$.

(g) $\theta_{m} \in \mathcal{D}_{R}^{e}\left[\theta_{1}, \theta_{p}, \ldots, \theta_{p^{e-1}}\right]$ for all $m<p^{e}$.

(h) $\mathcal{D}_{R}^{e}\left[\theta_{1}, \ldots, \theta_{p^{e-1}}\right] t=t \mathcal{D}_{R}^{e}\left[\theta_{1}, \ldots, \theta_{p^{e-1}}\right]$.

Proof. (a) and (b) are proven in [23, Lemma 4.1], and (c), (d), (e), (f) follow from (a) and [23, Lemma 4.1], (g) follows from [23, Remark 6.3] and (a). For (h) we argue along the lines of [23, Lemma 6.4]. The inclusion from right to left follows from (d) and (g). The other inclusion follows similarly using (f).

\section{$\S 2$. From Cartier modules to right $\mathcal{D}$-modules}

In this section, we recall the construction of the functor from Cartier modules to (right) $\mathcal{D}_{R}$-modules. 
Throughout we assume that $R$ is an $F$-finite regular ring and that all modules considered are finitely generated. By definition, a Cartier module $M$ is an $R$-module $M$ together with an $R$-linear map $\kappa: F_{*} M \rightarrow M$. This is equivalent to the data of an $R$-module and an $R$-linear map $C: M \rightarrow F^{!} M=$ $\operatorname{Hom}_{R}\left(F_{*} R, M\right)(C$ is just the adjoint of $\kappa$ - see [7, Proposition 2.18]). Iterating this map we obtain a directed system $M \rightarrow F^{!} M \rightarrow \cdots \rightarrow F^{e !} M$. We have

Proposition 2.1. Let $(M, \kappa)$ be a Cartier module. Then the limit $\mathcal{M}$ over the maps $M \rightarrow F^{e !} M$ yields an isomorphism $\mathcal{M} \rightarrow F^{!} \mathcal{M}$ which endows $\mathcal{M}$ with a right $\mathcal{D}_{R}$-module structure.

Proof. It is easy to see that the $C^{e}$ induce a map $\mathcal{M} \rightarrow F^{e !} \mathcal{M}$ which is an isomorphism for all $e \geqslant 0$. Each $F^{e !} M$ is naturally a right $\mathcal{D}_{R}^{e}$-module by premultiplication. This induces a right $\mathcal{D}_{R}$-module structure in the limit.

It is well known that if $R$ is smooth over a perfect field $k$ then the topdimensional differential forms $\omega_{R / k}$ are naturally equipped with a right $\mathcal{D}_{R^{-}}$ module structure and $\omega_{R / k}$ induces an equivalence between left and right $\mathcal{D}_{R}$-modules (see [3, Chapitre 1]).

If $k$ is only $F$-finite but not perfect then the situation is more complicated. We proceed as follows. Fix, once and for all, an isomorphism $k \rightarrow F^{!} k$. If $R$ is regular essentially of finite type over $k$ with structural morphism $f$ : Spec $R \rightarrow k$ then we set $\omega_{R}:=f^{!} k$ and we get an induced isomorphism $\omega_{R} \rightarrow$ $F^{!} \omega_{R}$ (note that $F$ is the absolute Frobenius morphism) ${ }^{2}$. This isomorphism endows $\omega_{R}$ with a right $\mathcal{D}_{R}$-module structure and after this choice one has an equivalence between right and left $\mathcal{D}_{R}$-modules that is obtained by tensoring with $\omega_{R}^{-1}$.

In particular, since direct limits commute with tensor products the category of $\mathcal{D}_{R}$-modules obtained in Proposition 2.1 (together with the fixed isomorphism) is equivalent to the category of unit $R[F]$-modules of Emerton and Kisin (see [5, Theorem 2.27]). Moreover, if we restrict the functor $M \mapsto \operatorname{colim}_{e} F^{e !} M$ to the category of minimal Cartier modules (or equivalently, if we descend it to Cartier crystals — see [7] and [8] for these notions) then it is also fully faithful.

Since test modules are naturally attached to Cartier modules it seems more natural to work with right- $\mathcal{D}_{R}$ modules when studying test module

\footnotetext{
${ }^{2}$ We are suppressing a shift here, but $f^{!} k$ is supported in a single degree and is an invertible sheaf.
} 
filtrations and Bernstein-Sato polynomials. In fact, using this approach we can employ the ordinary higher Euler operators (i.e. $t^{p^{i}} \partial_{t}^{\left[p^{i}\right]}$ instead of $\left.\partial_{t}^{\left[p^{i}\right]} t^{p^{i}}\right)$ and avoid a sign change in the definition of Bernstein-Sato polynomials compared to [23].

Note that the case Mustaţă considered in [23] corresponds in our setting to the Cartier module $\omega_{R}$ with the (chosen) isomorphism $\omega_{R} \rightarrow F^{!} \omega_{R}$. In terms of constructible sheaves on the étale site this corresponds, via the Riemann-Hilbert correspondence of [14] and [7, Theorem 5.15], to the case of the constant sheaf.

For left $\mathcal{D}_{R}^{e}$-modules one has, as a special case of Morita equivalence, an equivalence with $R$-Mod for all $e \geqslant 0$ (see e.g. [4, Proposition 3.8 and Corollary 3.10]). A similar statement holds for right modules:

Proposition 2.2. Let $R$ be an F-finite regular ring. Then the functor $F^{e !}(-)=\operatorname{Hom}_{R}\left(F_{*}^{e} R,-\right)$ induces an equivalence between Mod-R and Mod$\mathcal{D}_{R}^{e}$. Its inverse is given by $-\otimes_{\operatorname{End}_{R}\left(F_{*}^{e} R\right)} F_{*}^{e} R$. In particular, $F^{e !}$ reflects isomorphisms.

Proof. By the assumptions on $R$ we obtain that $F_{*}^{e} R$ is a finitely generated locally free $R$-module. Note that $F^{e^{!}} M=\operatorname{Hom}\left(F_{*}^{e} R, M\right)$ viewed as an $R$-module via the ring isomorphism $R \rightarrow F_{*}^{e} R$ and acting on the first factor is isomorphic to $F^{e *} M \otimes_{F_{*} R} F^{e !} R \cong M \otimes_{R} F^{e !} R$ by [7, Lemma 5.7]. Since $F^{e !} R=\operatorname{Hom}_{R}\left(F_{*}^{e} R, R\right)$ the claimed equivalence is just a case of Morita equivalence (cf. e.g. [18, Theorem 18.24]).

As $F^{e !}$ induces an equivalence it is fully faithful and hence reflects isomorphisms.

\section{§3. Bernstein-Sato polynomials}

In this section, we introduce our notion of Bernstein-Sato polynomial after transferring some results of Mustaţă in [23] to our right $\mathcal{D}$-module situation.

If $k$ is perfect and $\operatorname{Spec} R$ is smooth most of the results in this section follow formally once one observes that given a right $\mathcal{D}_{R}[t]$-module the operator $t^{p^{e}} \partial_{t}^{\left[p^{e}\right]}$ acts via $-\partial_{t}^{\left[p^{e}\right]} t^{p^{e}}$ on the left module obtained by tensoring with $\omega_{R[t] / k}^{-1}[3,1.3 .4]$.

LEMMA 3.1. Let $R$ be regular and $F$-finite and let $R[t]$ be the polynomial ring in one variable over $R$. Given a right $\mathcal{D}_{R[t]}^{e}$-module $M$, there is a unique 
decomposition as $\mathcal{D}_{R}^{e}$-modules

$$
M=\bigoplus_{i \in \mathbb{F}_{p}^{e}} M_{i},
$$

where for $1 \leqslant l \leqslant e$ the operator $\theta_{p^{l-1}}$ acts on $M_{i}$ via $i_{l}$. This decomposition is preserved by $\mathcal{D}_{R[t]}^{e}$-morphisms. The same statement holds for $\mathcal{D}_{R}^{e}\left[\theta_{1}, \ldots, \theta_{p^{e-1}}\right]$-modules.

Proof. This works similarly to [23, Proposition 4.2]. More precisely, one has

$$
\prod_{j=0}^{p-1}\left(\theta_{p^{e}}+j\right)=\prod_{j=1}^{p}\left(\theta_{p^{e}}+j\right)=0
$$

for all $e \geqslant 0$. Indeed, by Lemma 1.1 it suffices to show that $\left(\partial_{t}^{\left[p^{e}\right]}\right)^{p}=0$. This in turn follows from (b) since $\frac{p^{e+1} !}{\left(p^{e !}\right)^{p}}$ is divisible by $p$. Using this and the fact that $\left[\theta_{i}, \theta_{j}\right]=0$ the existence of such a decomposition follows. The remaining statements follow easily.

Given $M$ we refer to the decomposition of Lemma 3.1 as the eigenspace decomposition of $M$ (with respect to the Euler operators).

Note that if $M$ is a right $\mathcal{D}_{R[t]^{e}}^{e}$-module then it is in particular a right $\mathcal{D}_{R[t]^{-}}^{e-1}$ module. Hence, $M$ admits eigenspace decompositions with respect to $\mathcal{D}_{R[t]}^{e}$ and with respect to $\mathcal{D}_{R[t]}^{e-1}$ and these are compatible. That is, if $M_{\left(i_{1}, \ldots, i_{e-1}\right)}$ is an eigenspace for the $\theta_{p^{l}-1}$ with $1 \leqslant l \leqslant e-1$ then

$$
M_{\left(i_{1}, \ldots, i_{e-1}\right)}=\bigoplus_{j \in \mathbb{F}_{p}} M_{\left(i_{1}, \ldots, i_{e-1}, j\right)}
$$

is an eigenspace decomposition with respect to the $\theta_{p^{l}-1}$ for $1 \leqslant l \leqslant e$. Again, a similar statement holds for right $\mathcal{D}_{R}^{e}\left[\theta_{1}, \ldots, \theta_{p^{e-1}}\right]$-modules.

Given a morphism $f: \operatorname{Spec} S \rightarrow \operatorname{Spec} R$ of regular schemes and a right $\mathcal{D}_{S}$ module $M$ one defines the pushforward $f_{+} M$ as $f_{*}\left(M \otimes_{\mathcal{D}_{S}} S\right) \otimes_{R} \mathcal{D}_{R}$. We thus have a natural map $f_{*} M \rightarrow f_{+} M$. By abuse of notation we denote the image of $f_{*} M$ under this map again by $f_{*} M$. Similarly, we define the pushforward $f_{+} M$ for a right $\mathcal{D}_{S}^{e}$-module as $f_{*}\left(M \otimes_{\mathcal{D}_{S}^{e}} S\right) \otimes_{R} \mathcal{D}_{R}^{e}$.

Our next goal is to describe this natural map in the setting where we identify $\mathcal{D}_{R}^{e}$ with $\operatorname{End}_{R}\left(F_{*}^{e} R\right)$. We first need a

Lemma 3.2. Let $R$ be regular and $F$-finite and $M$ a (right) $R$-module. Then $F_{*}^{e} M \otimes_{F_{*}^{e} R} \operatorname{End}_{R}\left(F_{*}^{e} R\right) \rightarrow \operatorname{Hom}_{R}\left(F_{*}^{e} R, M\right), m \otimes \varphi \mapsto[r \mapsto m \varphi(r)]$ is an isomorphism of $\operatorname{End}_{R}\left(F_{*}^{e} R\right)$-modules. 
Proof. The module structure on $F_{*}^{e} M \otimes_{F_{*} R} \operatorname{End}_{R}\left(F_{*}^{e} R\right)$ is given by multiplication from the right and the one on $\operatorname{Hom}_{R}\left(F_{*}^{e} R, M\right)$ is given by premultiplication. Bijectivity is local so that we may assume that $F_{*}^{e} R$ is a free $R$-module (use [17, Theorem 2.1]). Fix a basis $b_{1}, \ldots, b_{n}$ and let $\delta_{i}: F_{*}^{e} R \rightarrow F_{*}^{e} R$ be the projection onto the $i$ th basis vector. We define a map $\operatorname{Hom}_{R}\left(F_{*}^{e} R, M\right) \rightarrow F_{*}^{e} M \otimes_{F_{*}^{e} R} \operatorname{End}_{R}\left(F_{*}^{e} R\right), \varphi \mapsto \sum_{i} \varphi\left(b_{i}\right) \otimes \delta_{i}$ which is a two-sided inverse.

Proposition 3.3. Let $f: \operatorname{Spec} S \rightarrow \operatorname{Spec} R$ be a morphism of regular $F$-finite schemes and let $M$ be an $S$-module. Let $\operatorname{Hom}_{S}\left(F_{*}^{e} S, M\right)$ be a right $\mathcal{D}_{S}^{e}$-module via the action on the first factor. Then $f_{+} \operatorname{Hom}_{S}\left(F_{*}^{e} S, M\right)$ is naturally isomorphic to $F^{e !} f_{*} M$. Under this identification the natural map

$$
f_{*} \operatorname{Hom}_{S}\left(F_{*}^{e} S, M\right) \rightarrow f_{+} \operatorname{Hom}_{S}\left(F_{*}^{e} S, M\right)
$$

is given by the composition of the canonical maps

$$
f_{*} \operatorname{Hom}_{S}\left(F_{*}^{e} S, M\right) \rightarrow \operatorname{Hom}_{R}\left(f_{*} F_{*}^{e} S, f_{*} M\right) \rightarrow \operatorname{Hom}_{R}\left(F_{*}^{e} f_{*} S, f_{*} M\right)
$$

with the map

$$
\operatorname{Hom}_{R}\left(F_{*}^{e} f_{*} S, f_{*} M\right) \rightarrow \operatorname{Hom}_{R}\left(F_{*}^{e} R, f_{*} M\right), \quad \varphi \mapsto \varphi \circ F_{*}^{e}\left(f^{\#}\right) .
$$

Proof. By definition the pushforward $f_{+} \operatorname{Hom}_{S}\left(F_{*}^{e} S, M\right)$ is given as

$$
F_{*}^{e} f_{*}\left(\operatorname{Hom}_{S}\left(F_{*}^{e} S, M\right) \otimes_{\operatorname{End}_{S}\left(F_{*}^{e} S\right)} F_{*}^{e} S\right) \otimes_{F_{*}^{e} R} \operatorname{End}_{R}\left(F_{*}^{e} R\right) .
$$

Note that the right $F_{*}^{e} R$-module structure on the term $F_{*}^{e} f_{*}(\ldots)$ is given by $(\psi \otimes s) \cdot r=\psi \otimes s f(r)$. By Proposition 2.2 the whole expression is isomorphic to $F_{*}^{e} f_{*} M \otimes_{F_{*}^{e} R} \operatorname{End}_{R}\left(F_{*}^{e} R\right)$. Using Lemma 3.2 above we obtain that $F_{*}^{e} f_{*} M \otimes_{F_{*}^{e} R} \operatorname{End}_{R}\left(F_{*}^{e} R\right) \rightarrow \operatorname{Hom}_{R}\left(F_{*}^{e} R, f_{*} M\right)$ is an isomorphism. One readily checks that the natural map is given by the formula above.

Next we show that this isomorphism is compatible with direct limits. We first need a general Lemma.

LEMma 3.4. Let $I$ be a directed system and let $M_{i}, N_{i}$ be $R_{i}$-modules such that $M_{i}, N_{i}$ and $R_{i}$ are filtered by $I$. Write $M=\operatorname{colim}_{i} M_{i}, N=$ $\operatorname{colim}_{i} N_{i}$ and $R=\operatorname{colim}_{i} R_{i}$. Then one has an isomorphism $\operatorname{colim}_{i}\left(M_{i} \otimes_{R_{i}}\right.$ $\left.N_{i}\right) \rightarrow M \otimes_{R} N$. 
Proof. We have $R_{i}$-bilinear maps $M_{i} \times N_{i} \rightarrow M \otimes_{R} N$ which induce, by the universal properties of the limit and the tensor product, an $R$-linear map $\operatorname{colim}_{i} M_{i} \otimes_{R_{i}} N_{i} \rightarrow M \otimes N,\left[m_{i} \otimes n_{i}\right] \mapsto\left[m_{i}\right] \otimes\left[n_{i}\right]$.

On the other hand, the maps $M_{i} \times N_{i} \rightarrow \operatorname{colim}_{i} M_{i} \otimes_{R_{i}} N_{i},\left(m_{i}, n_{i}\right) \mapsto$ $\left[m_{i} \otimes n_{i}\right]$ induce an $R$-bilinear map $M \times N \rightarrow \operatorname{colim}_{i} M_{i} \otimes_{R_{i}} N_{i}$. This in turn induces an $R$-linear map $M \otimes N \rightarrow \operatorname{colim}_{i} M_{i} \otimes_{R_{i}} N_{i}$ which is an inverse to the map constructed above.

Proposition 3.5. Let $f: \operatorname{Spec} S \rightarrow \operatorname{Spec} R$ be a morphism of regular $F$ finite schemes and assume that $(M, \kappa)$ is a Cartier module on Spec $S$ and denote its limit over the $C^{e}$ by $\mathcal{M}$. Then $f_{+} \mathcal{M}$ is naturally isomorphic to $\operatorname{colim} F^{e !} f_{*} M \cong \operatorname{colim} f_{+} F^{e !} M$.

Proof. The first claimed isomorphism is obtained by applying Lemma 3.4 twice.

For the second claimed isomorphism note that by Proposition 3.3 we have isomorphisms $f_{+} F^{e !} M \rightarrow F^{e !} f_{*} M$. Moreover, we have a commutative diagram

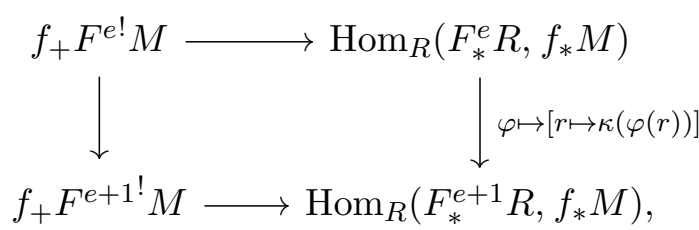

where the left vertical map is given by tensoring $\varphi \mapsto[s \mapsto \kappa(\varphi(s))]$ with the natural maps $F_{*}^{e} S \rightarrow F_{*}^{e+1} S$ and $\mathcal{D}_{S}^{e} \rightarrow \mathcal{D}_{S}^{e+1}$.

Lemma 3.6. Let $R$ be regular and $F$-finite and $f \in R$. Denote by $\gamma$ : Spec $R \rightarrow$ Spec $R[t]$ the graph embedding along $f$ and let $M$ be a right $\mathcal{D}_{R^{-}}$ module. Then the quotient

$$
N:=\left(\gamma_{*} M\right) \mathcal{D}_{R}^{e}\left[\theta_{1}, \theta_{p}, \ldots, \theta_{p^{e-1}}\right] /\left(\gamma_{*} M\right) \mathcal{D}_{R}^{e}\left[\theta_{1}, \theta_{p}, \ldots, \theta_{p^{e-1}}\right] t
$$

is a right $\mathcal{D}_{R}^{e}\left[\theta_{1}, \theta_{p}, \ldots, \theta_{p^{e-1}}\right]$-module.

Proof. The claim follows from Lemma 1.1 (i) and $\left(\gamma_{*} M\right) t=\gamma_{*}(M f)$.

Definition 3.7. With the notation of Lemma 3.6 let $\Gamma_{f}^{e} \subseteq\{0, \ldots, p-$ $1\}^{e}$ be the set of those $i=\left(i_{1}, \ldots, i_{e}\right) \in \mathbb{F}_{p}^{e}$ for which the eigenspace $N_{i}$ of $N$ (as constructed in Lemma 3.1) is nontrivial. Then we define the eth 
Bernstein-Sato polynomial of $M$ as

$$
b_{M, f}^{e}(s)=\prod_{i \in \Gamma_{f}^{e}}\left(s-\left(\frac{i_{e}}{p}+\cdots+\frac{i_{1}}{p^{e}}\right)\right) \in \mathbb{Q}[s],
$$

where we lift ${ }^{3}$ elements of $\mathbb{F}_{p}=\{0, \ldots, p-1\}$ to $\mathbb{Z}$.

Note that since we are working with right modules rather then left modules we do not need to invert the sign of the eigenvalues as in [23]. More precisely, Mustaţă considers the action of $\partial^{\left[p^{l}\right]} t^{p^{l}}$ on the left $\mathcal{D}_{R}^{e}\left[\vartheta_{1}, \ldots, \vartheta_{p^{e-1}}\right]$-module

$$
\mathcal{D}_{R}^{e}\left[\vartheta_{1}, \ldots, \vartheta_{p^{e-1}}\right] \gamma_{*} R / t \mathcal{D}_{R}^{e}\left[\vartheta_{1}, \ldots, \vartheta_{p^{e-1}}\right] \gamma_{*} R
$$

and if $\left(i_{1}, \ldots, i_{e}\right)$ is an eigenvalue for the left action then $\frac{-i_{e}}{p}+\cdots+\frac{-i_{1}}{p^{e}}$ is encoded as a zero in a Bernstein-Sato polynomial. As pointed out at the beginning of Section 3 these Bernstein-Sato polynomials coincide with the one defined in 3.7 provided $R$ is smooth over a perfect field $k$. In fact, Theorem 5.4 and [23, Theorem 6.7] show that the polynomials coincide for $R$ regular essentially of finite type over an $F$-finite field and $e \gg 0$.

REMARK 3.8. We comment on our definition of Bernstein-Sato polynomial and its relation to the definition over the complex numbers. Let $X=\mathbb{A}_{\mathbb{C}}^{n+1}$. Then $\mathcal{D}_{X}$ is just the Weyl algebra $\mathbb{C}\left[x_{1}, \ldots, x_{n+1}, \partial_{1}, \ldots, \partial_{n+1}\right]$ with the usual relation $\left[\partial_{i}, x_{j}\right]=\delta_{i j}$. Assume that the hypersurface is given by $t=x_{n+1}$. Then for a regular holonomic quasi-unipotent $\mathcal{D}_{X}$-module $M$ the $V$-filtration along $t$ is a decreasing $\mathbb{Q}$-indexed filtration with certain properties (see [11] for a definition).

In particular, one has (cf. [24, Proposition 2.1.7]) $V^{k} M=V^{k}\left(\mathcal{D}_{R}\right) V^{0}(M)$ for $k \leqslant 0$ but $V^{k} M=V^{k-1}\left(\mathcal{D}_{R}\right) V^{1}(M)$ only for $k \geqslant 1 .{ }^{4}$ Here $V^{k}\left(\mathcal{D}_{X}\right)$ is the $V$-filtration on $\mathcal{D}_{X}$ which is given by

$$
V^{k}\left(\mathcal{D}_{X}\right)=t^{k} V^{0}\left(\mathcal{D}_{X}\right) \text { for } k \geqslant 1
$$

and by

$$
V^{k}\left(\mathcal{D}_{X}\right)=V^{0}\left(\mathcal{D}_{X}\right) \mathcal{D}_{X,-k} \quad \text { for } k \leqslant-1
$$

\footnotetext{
${ }^{3}$ Here and elsewhere we always view $\mathbb{F}_{p}$ as $\{0, \ldots, p-1\}$ and confuse elements in $\mathbb{F}_{p}$ with a lift whenever this is convenient.

${ }^{4}$ Note that following [11] we have inverted signs here compared to [24].
} 
where $\mathcal{D}_{X,-k}$ are the differential operators of order $\leqslant-k$. Finally, $V^{0}\left(\mathcal{D}_{X}\right)$ is given by $\left\{\sum_{n \geqslant m} f(x) \partial_{x}^{\alpha} t^{n} \partial_{t}^{m} \mid f \in \mathbb{C}\left[x_{1}, \ldots, x_{n}\right]\right\}$.

The Bernstein-Sato polynomial of $M$ is now defined as the monic minimal polynomial $b \in \mathbb{C}[s]$ such that $b\left(t \partial_{t}+k\right) V^{k} M \subseteq V^{k+1} M$ for all $k \in \mathbb{Z}$. By the above it is actually sufficient to construct a Bernstein-Sato polynomial that satisfies the above identity for $k=-1,0,1$.

Since in characteristic $p>0$ the Briançon-Skoda theorem [6, Theorem 4.21] always yields $f^{n} \tau\left(M, f^{t-n}\right)=\tau\left(M, f^{t}\right)$ for $t \geqslant n$ our definition can be seen as an analog of the one over the complex numbers since we only need to control the range $k=0$.

\section{$\S 4$. Test modules and $\mathcal{D}$-modules}

In this section, we relate test modules of a Cartier module $M$ over a regular $F$-finite ring $R$ with certain right $\mathcal{D}_{R}^{e}$ submodules of $F^{e !} M$. First, we need several technical lemmata concerning test modules.

LEMma 4.1. Let $R$ be essentially of finite type over an F-finite field, $(M, \kappa)$ an $F$-pure coherent Cartier module, $t \in \mathbb{Q} \geqslant 0$ and let $f$ be an $M$ regular element. Then one has $\kappa^{e} f^{\left\lceil t p^{e}\right\rceil} f^{l} M \subseteq \kappa^{e+1} f^{\left\lceil t p^{e+1}\right\rceil} f^{l} M$ for all $l \geqslant 0$. In particular, for $e \gg 0$ depending on $l$ equality holds.

Proof. First of all, for any $l$ we have

$$
\kappa^{n} f^{l} M \supseteq \kappa^{n} f^{l p} M=\kappa^{n-1} f^{l} \kappa M=\kappa^{n-1} f^{l} M,
$$

where we used that $\kappa$ is surjective since $M$ is $F$-pure.

Next, we have

$$
\kappa f^{\left\lceil t p^{e+1}\right\rceil} f^{l} M \supseteq \kappa\left(f^{\left\lceil t p^{e}\right\rceil}\right)^{p} f^{l} M=f^{\left\lceil t p^{e}\right\rceil} \kappa f^{l} M \supseteq f^{\left\lceil t p^{e}\right\rceil} f^{l} M,
$$

where we used the previous observation with $n=1$ for the last inclusion. Applying $\kappa^{e}$ on both sides yields the claimed inclusion. Since $M$ is coherent the ascending chain $\kappa^{e} f^{\left\lceil t p^{e}\right\rceil} f^{l} M$ stabilizes.

REMARK 4.2. With the notation of Lemma 4.1 if $t=\frac{m}{p^{s}}$ and $n \geqslant s$ is such that $\kappa^{n} f^{l} M=M$ then equality holds for any $e \geqslant 2 n$. Indeed,

$$
\kappa^{e} f^{m p^{e-s}} f^{l} M=\kappa^{e}\left(f^{m p^{n-s}}\right)^{p^{e-n}} f^{l} M=\kappa^{n} f^{m p^{n-s}} \kappa^{e-n} f^{l} M=\kappa^{n} f^{m p^{n-s}} M
$$

which is independent of $e$.

If $R$ is a polynomial ring and $M$ is given explicitly by a presentation $R^{a} \rightarrow R^{b} \rightarrow M$ then we expect that it should be possible to determine $e$ explicitly for a given hypersurface $f$. 
LEMMA 4.3. Let $R$ be essentially of finite type over an F-finite field, let $(M, \kappa)$ be an $F$-regular coherent Cartier module, $t \in \mathbb{Z}\left[\frac{1}{p}\right]$ and let $f$ be an $M$-regular element. Consider the Cartier algebra $\mathcal{C}$ generated in degree $e \geqslant 1$ by $\kappa^{e} f^{\left\lceil t p^{e}\right\rceil}$. Then $\underline{M}_{\mathcal{C}}=\kappa^{e} f^{\left\lceil t p^{e}\right\rceil} M$ for all $e \gg 0$.

Proof. The calculation

$$
\kappa^{a} f^{\left\lceil t p^{a}\right\rceil} \kappa^{a^{\prime}} f^{\left\lceil t p^{a^{\prime}}\right\rceil}=\kappa^{a+a^{\prime}} f^{\left\lceil t p^{a}\right\rceil p^{a^{\prime}}} f^{\left\lceil t p^{a^{\prime}}\right\rceil}=\kappa^{a+a^{\prime}} f^{\left\lceil t p^{a+a^{\prime}}\right\rceil} f^{r}
$$

with $0 \leqslant r=\left\lceil t p^{a^{\prime}}\right\rceil+\left\lceil t p^{a}\right\rceil p^{a^{\prime}}-\left\lceil t p^{a+a^{\prime}}\right\rceil$ shows that $\mathcal{C}$ is in indeed a Cartier algebra. Applying Lemma 4.1 with $l=0$ shows that $\kappa^{e} f^{\left\lceil t p^{e}\right\rceil} M \subseteq$ $\kappa^{e+1} f^{\left\lceil t p^{e+1}\right\rceil} M$ (with equality for $e \gg 0$ ) so that for all $e \gg 0$ we have $\kappa^{e} f^{\left\lceil t p^{e}\right\rceil} M=\mathcal{C}_{+} M$.

By [6, Proposition 2.13 and Corollary 2.14], one has $\underline{M}_{\mathcal{C}}=\left(\mathcal{C}_{+}\right)^{h} M$ for all $h \gg 0$. Fix such an $h$ and $e$ as above. Then the inclusion from left to right follows by the same argument as above.

For the other inclusion we have to prove that $\mathcal{C}_{+} \kappa^{e} f^{\left\lceil t p^{e}\right\rceil} M \supseteq \kappa^{e} f^{\left\lceil t p^{e}\right\rceil} M$ for $e \gg 0$. We use the assumption on $t$ and write $t=\frac{m}{p^{s}}$ with $m \in \mathbb{Z}$ and assume that $e \geqslant s$. We consider elements of the form $\kappa^{e^{\prime}} f\left\lceil t p^{e^{\prime}}\right\rceil \in \mathcal{C}_{+}$. For $e^{\prime} \geqslant e$ we compute

$$
\kappa^{e^{\prime}} f^{m p^{e^{\prime}-s}} \kappa^{e} f^{m p^{e-s}} M=\kappa^{e} f^{m p^{e-s}} \kappa^{e^{\prime}-e} \kappa^{e} f^{m p^{e-s}} M=\kappa^{e} f^{m p^{e-s}} M,
$$

where for the last equality we used the $F$-regularity of $(M, \kappa)$ (see $[25$, Proposition 5.2] and Lemma 4.1) possibly choosing a larger $e^{\prime}$.

Next, we prove a variant of [10, Lemma 2.1] for modules.

LEMMA 4.4. Let $R$ be essentially of finite type over an F-finite field. Let $(M, \kappa)$ be an $F$-regular coherent Cartier module, $f \in R$ a nonzero divisor on $M$ and $t \in \mathbb{Z}\left[\frac{1}{p}\right]$. Then for all $e \gg 0$ we have $\tau\left(M, f^{t}\right)=\kappa^{e}\left(f^{t p^{e}} M\right)$.

Proof. First of all, note that $M_{f}$ is $F$-regular with respect to the Cartier algebra $\mathcal{C}$ generated by the $\kappa^{e} f^{\left\lceil t p^{e}\right\rceil}$. By [6, Theorem 3.11], we thus have

$$
\tau\left(M, f^{t}\right)=\sum_{n \geqslant 1} \mathcal{C}_{n} f \underline{M}_{\mathcal{C}} .
$$

By Lemma 4.3 we may replace $\underline{M}_{\mathcal{C}}$ by $\kappa^{e} f^{t p^{e}} M$ for any sufficiently large $e$. As seen in the proof of Lemma 4.3 we have $\mathcal{C}_{n}=\kappa^{n} f^{\left\lceil t p^{n}\right\rceil} R$. 
We compute

$$
\kappa^{n} f^{\left\lceil t p^{n}\right\rceil} f \kappa^{e} f^{t p^{e}} M=\kappa^{n+e} f^{\left\lceil t p^{n}\right\rceil p^{e}} f^{(t+1) p^{e}} M \subseteq \kappa^{n+e} f^{\left\lceil t p^{n+e}\right\rceil} f^{(t+1) p^{e}} M
$$

and for $t p^{n} \in \mathbb{Z}$ equality holds. In particular, using Lemma 4.1 we obtain that

$$
\sum_{n \geqslant 1} \kappa^{n} f^{\left\lceil t p^{n}\right\rceil} f \kappa^{e} f^{t p^{e}} M=\kappa^{n} f^{t p^{n}} f \kappa^{e} f^{t p^{e}} M \quad \text { for some } n \gg 0
$$

Finally, we have for $n \gg 0$ and suitable $e^{\prime}$

$$
\kappa^{n} f^{t p^{n}} f \kappa^{e} f^{t p^{e}} M=\kappa^{n+e-e^{\prime}} f^{t p^{n+e-e^{\prime}}} \kappa^{e^{\prime}} f^{(t+1) p^{e}} M=\kappa^{n+e-e^{\prime}} f^{t p^{n+e-e^{\prime}}} M,
$$

where the last equality is due to the $F$-regularity of $(M, \kappa)$.

REMARK 4.5. In particular, if $t=\frac{m}{p^{s}} \in \mathbb{Z}\left[\frac{1}{p}\right]$ we may write $t=\frac{m p^{e-s}}{p^{e}}$ so that $\tau\left(M, f^{t}\right)=\kappa^{e}\left(f^{m^{\prime}} M\right)$, where $m^{\prime}=m p^{e-s}$.

Also note that by right continuity (i.e. $\tau\left(M, f^{t}\right)=\tau\left(M, f^{t+\varepsilon}\right)$ for small $\varepsilon>0$ ) we may always assume that $t \in \mathbb{Z}\left[\frac{1}{p}\right]$ if we want to compute test modules.

Given a $\kappa$-module $M$ we have a natural map (the adjoint of $\kappa) C: M \rightarrow$ $F^{!} M, m \mapsto(r \mapsto \kappa(r m))$ and if $N$ is an $R$-submodule of $M$ we may consider the right $\mathcal{D}_{R}^{e}$-submodule of $F^{e !} M$ generated by $N$ which by definition is $C^{e}(N) \cdot \mathcal{D}_{R}^{e}$.

LEMma 4.6. Let $R$ be regular and $F$-finite. Let $M$ be a coherent $\kappa$ module and let $N$ be an R-submodule of $M$. Then $F^{e !} \kappa^{e}\left(F_{*}^{e} N\right)$ is the right $\mathcal{D}_{R}^{e}$-submodule of $F^{e !} M$ generated by the image of $N$ in $F^{e !} M$.

Proof. Clearly, $F^{e !} \kappa^{e}\left(F_{*}^{e} N\right)$ is a right $\mathcal{D}_{R^{-}}^{e}$ submodule of $F^{e !} M$. So one inclusion is dealt with once we show that $C^{e}(N) \subseteq F^{e !} \kappa^{e}\left(F_{*}^{e} N\right)$. If $n \in N$ then $C^{e}(n): F_{*}^{e} R \rightarrow M, r \mapsto \kappa^{e}(r n)$ and $C^{e}(r n) \in \kappa^{e}\left(F_{*}^{e} N\right)$ for all $r \in F_{*}^{e} R$.

For the other inclusion we may assume that $R$ is local. Hence, $F_{*}^{e} R$ is free of rank $s=p^{e \operatorname{dim} R}$ since $R$ is regular and $F$-finite (see [17, Theorem 2.1]). Fix a basis $b_{1}, \ldots, b_{s}$ of $F_{*}^{e} R$ and let $\varphi: F_{*}^{e} R \rightarrow \kappa^{e}\left(F_{*}^{e} N\right)$ be an element of $F^{e !} \kappa^{e}\left(F_{*}^{e} N\right)$. Each $\varphi\left(b_{i}\right)$ is of the form $\kappa^{e}\left(n_{i}\right)$ for some $n_{i} \in N$. We then can write $\varphi$ as $\sum_{i=1}^{s} \psi_{i} \circ p_{i}$, where $p_{i}: F_{*}^{e} R \rightarrow F_{*}^{e} R, p_{i}\left(b_{j}\right)=\delta_{i j}$ and $\psi_{i}(r)=$ $\kappa^{e}\left(r n_{i}\right)$. Then $p_{i} \in \mathcal{D}_{R}^{e}$ and $\psi_{i} \in C^{e}(N)$ as desired. 
ExAmple 4.7. Let $M$ be a Cartier module on $R$ and let $\gamma:$ Spec $R \rightarrow$ Spec $R[t]$ be the graph embedding along $f$. Then one has, in general, a proper inclusion $\gamma_{*}\left(C^{e}(M)\right) \mathcal{D}_{R}^{e} \subseteq \gamma_{*}\left(C^{e}(M) \mathcal{D}_{R}^{e}\right) \mathcal{D}_{R}^{e}$. Here we let $\mathcal{D}_{R}^{e}$ act via the inclusion $\mathcal{D}_{R}^{e} \rightarrow \mathcal{D}_{R[t]}^{e}$. Also note that $C^{e}(M) \mathcal{D}_{R}^{e}=F^{e !} M$.

As an example for this let $R=k[y]$ and $M=R$ with twisted Cartier structure $\kappa \cdot y$, where $\kappa\left(y^{i}\right)=\delta_{i(p-1)}$ for $0 \leqslant i \leqslant p-1$. Then $F^{e !} R$ contains $\kappa^{e}$ and we claim that this is not contained in $\gamma_{*}\left(C^{e}(M)\right) \mathcal{D}_{R}^{e}$. Indeed, any element $\psi$ in $\gamma_{*}\left(C^{e}(M)\right) \mathcal{D}_{R}^{e}$ is of the form

$$
\psi\left(y^{i} t^{j}\right)=\sum_{l=0}^{s} \kappa\left(y^{\frac{p^{e}-1}{p-1}} r_{l} P_{l}\left(y^{i}\right) y^{j}\right)
$$

where $P_{l} \in \mathcal{D}_{R}^{e}$ and we used that $(\kappa y)^{e}=\kappa^{e} y^{1+p+\cdots+p^{e-1}}$. Here $\kappa^{e}$ is given by acting on basis elements $y^{i} t^{j} \mapsto 1$ if $i+j=p^{e}-1$ and 0 else. To see the claim let now $i=0$ and $j=p^{e}-1$. Then we can write $\psi\left(t^{p^{e}-1}\right)$ as $y \cdot \sum \ldots$ and clearly this cannot evaluate to 1 . Hence, the inclusion is strict.

Combining the two previous lemmata we obtain the following

COROLlary 4.8. Let $R$ be regular and essentially of finite type over an $F$-finite field. Let $(M, \kappa)$ be an F-regular Cartier module and let $f \in R$ be a nonzero divisor on $M$ and $t \in \mathbb{Z}\left[\frac{1}{p}\right]$. Then for $e \gg 0$ and $t=\frac{m}{p^{e}}$ we have $F^{e !} \tau\left(M, f^{t}\right)=C^{e}\left(f^{m} M\right) \cdot \mathcal{D}_{R}^{e}$.

Proof. Immediate from Lemma 4.4 and Lemma 4.6.

\section{$\S 5$. Test modules and Bernstein-Sato polynomials}

In this section, we prove the main result of this paper. That is, we show that the roots of the Bernstein-Sato polynomials for $e$ sufficiently large are precisely the $F$-jumping numbers in the range $(0,1]$ of the test module filtration.

Recall that given a morphism $\gamma: \operatorname{Spec} R \rightarrow \operatorname{Spec} R[t]$ and a right $\mathcal{D}_{R^{-}}^{e}$ module $M$ we denote the image of the natural map $\gamma_{*} M \rightarrow \gamma_{+} M$ again by $\gamma_{*} M$. In particular, $\gamma_{*} M$ is then contained in $\operatorname{Hom}_{R[t]}\left(F_{*}^{e} R[t], \gamma_{*} M\right.$ ) (see Proposition 3.3).

Lemma 5.1. Let $R$ be a regular F-finite ring and $(M, \kappa)$ a Cartier module with adjoint $C$. Let $f \in R$ and denote by $\gamma: \operatorname{Spec} R \rightarrow \operatorname{Spec} R[t]$ the graph embedding along $f$. Then we have an eigenspace decomposition

$$
\left(\gamma_{*} C^{e}(M)\right) \mathcal{D}_{R}^{e}\left[\theta_{1}, \ldots, \theta_{p^{e-1}}\right]=\bigoplus_{i \in \mathbb{F}_{p}^{e}}\left(\gamma_{*} C^{e}(M)\right) \mathcal{D}_{R}^{e} \circ \pi_{i},
$$


where

$$
\pi_{i}: F_{*}^{e} R[t] \rightarrow F_{*}^{e} R[t], \quad \pi_{i}\left(r t^{j}\right)= \begin{cases}r t^{j} & j=\sum_{l=1}^{e} i_{l} p^{l-1} \\ 0 & \text { else }\end{cases}
$$

is the projection onto the eigenspace.

Proof. We denote $\left(\gamma_{*} C^{e}(M)\right) \mathcal{D}_{R}^{e}\left[\theta_{1}, \ldots, \theta_{p^{e-1}}\right]$ by $N$ and its eigenspaces by $N_{i}$. First of all, note that Lucas' theorem shows that $\pi_{i}$ is the projection onto the eigenspace $i_{1}, \ldots, i_{e}$ for the $\theta_{1}, \ldots, \theta_{p^{e-1}}$.

Assume that $\varphi \in N_{i}$. Then viewing $\varphi$ as an element of $\operatorname{Hom}_{R[t]}\left(F_{*}^{e} R[t], \gamma_{*} M\right)$ we have $\varphi\left(r t^{m}\right)=0$ for $m \neq i_{1}+i_{2} p+\cdots+i_{e} p^{e-1}$ so that $\varphi$ factors through $\pi_{i}$.

In the other direction note that given $\varphi \in N$ we have $\varphi=$ $\sum_{i \in \mathbb{F}_{p}^{e}} \varphi \circ \pi_{i}$ which is a decomposition of $\varphi$ in the ambient module $\left(\gamma_{*} F^{!} M\right) \mathcal{D}_{R}^{e}\left[\theta_{1}, \ldots, \theta_{p^{e-1}}\right]$ as eigenvectors. Since this decomposition is preserved by morphisms (see Lemma 3.1) the above has to be the decomposition of $\varphi$ in $N$ as well.

Proposition 5.2. Let $R$ be a regular $F$-finite ring and $(M, \kappa)$ a Cartier module with adjoint $C$. Let $f \in R$ and denote by $\gamma: \operatorname{Spec} R \rightarrow$ Spec $R[t]$ the graph embedding along $f$. Then the $\left(i_{1}, \ldots, i_{e}\right)$-eigenspace of $\left(\gamma_{*} C^{e}(M)\right) \mathcal{D}_{R}^{e}\left[\theta_{1}, \ldots, \theta_{p^{e-1}}\right]$ is isomorphic to $C^{e}\left(f^{i_{1}+i_{2} p+\cdots i_{e} p^{e-1}} M\right) \cdot \mathcal{D}_{R}^{e}$ as a right $\mathcal{D}_{R}^{e}$-module.

Proof. We write $m=i_{1}+i_{2} p+\cdots+i_{e} p^{e-1}$. Clearly, the right $\mathcal{D}_{R^{-}}^{e}$ submodule $F_{*}^{e} R \cdot t^{m}$ of $F_{*}^{e} R[t]$ is isomorphic to $F_{*}^{e} R$ by sending $t^{m}$ to 1 . In particular, we have a commutative diagram

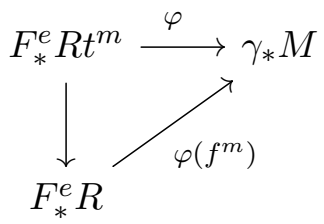

for any element $\varphi$ of the eigenspace. This induces the desired isomorphism of right $\mathcal{D}_{R}^{e}$-modules.

Corollary 5.3. The quotient

$$
\left(\gamma_{*} C^{e}(M)\right) \mathcal{D}_{R}^{e}\left[\theta_{1}, \ldots, \theta_{p^{e}-1}\right] /\left(\gamma_{*} C^{e}(f M)\right) \mathcal{D}_{R}^{e}\left[\theta_{1}, \ldots, \theta_{p^{e}-1}\right]
$$


is a right $\mathcal{D}_{R}^{e}\left[\theta_{1}, \ldots, \theta_{p^{e}-1}\right]$-module and the $\left(i_{1}, \ldots, i_{e}\right)$-eigenspace of the quotient is isomorphic to

$$
C^{e}\left(f^{i_{1}+i_{2} p+\cdots+i_{e} p^{e-1}} M\right) \cdot \mathcal{D}_{R}^{e} / C^{e}\left(f^{1+i_{1}+i_{2} p+\cdots+i_{e} p^{e-1}} M\right) \cdot \mathcal{D}_{R}^{e}
$$

as a right $\mathcal{D}_{R}^{e}$-module.

Proof. The first claim is just Lemma 3.6. Since the eigenspace decomposition is preserved by the canonical projection we get the desired isomorphism on the quotient by Proposition 5.2.

Following [23] we introduce some notation. Namely, given $\lambda \in(0,1]$ we can write it uniquely as

$$
\lambda=\sum_{i \geqslant 1} \frac{c_{i}(\lambda)}{p^{i}}
$$

with all $c_{i}(\lambda) \in\{0, \ldots, p-1\}$, and such that infinitely many of them are nonzero. Moreover, one obtains for every $e \geqslant 1$ that

$$
\sum_{i=1}^{e} \frac{c_{i}(\lambda)}{p^{i}}=\frac{\left\lceil\lambda p^{e}\right\rceil-1}{p^{e}} .
$$

We are now ready to state and prove our main result:

THEOREM 5.4. Let $R$ be regular essentially of finite type over an $F$-finite field. Let $(M, \kappa)$ be an $F$-regular Cartier module and $f \in R$ a nonzero divisor on $M$. The roots of the Bernstein-Sato polynomials $b_{M, f}^{e}(s)$ are given for $e$ sufficiently large by $\frac{\left\lceil\lambda p^{e}\right\rceil-1}{p^{e}}$, where $\lambda$ varies over the $F$-jumping numbers of the test module filtration $\tau\left(M, f^{t}\right)$ for $t \in(0,1]$.

Proof. By definition $\lambda \in(0,1]$ is an $F$-jumping number if and only if for all $e \gg 0$ we have

$$
\tau\left(M, \kappa, f^{\frac{\left\lceil\lambda p^{e}\right\rceil-1}{p^{e}}}\right) \neq \tau\left(M, \kappa, f^{\frac{\left\lceil\lambda p^{e}\right\rceil}{p^{e}}}\right) .
$$

Using the fact that $F^{e !}$ is fully faithful (Proposition 2.2) this inequality is equivalent to

$$
F^{e !} \tau\left(M, \kappa, f^{\frac{\left\lceil\lambda p^{e}\right\rceil-1}{p^{e}}}\right) \neq F^{e !} \tau\left(M, \kappa, f^{\frac{\left\lceil\lambda p^{e}\right\rceil}{p^{e}}}\right)
$$

for all $e \gg 0$. We write $\left\lceil\lambda p^{e}\right\rceil-1=i_{1}+i_{2} p+\cdots+i_{e} p^{e-1}$. Then by Corollary 4.8 the above means that

$$
C^{e}\left(f^{i_{1}+i_{2} p+\cdots+i_{e} p^{e-1}} M\right) \cdot \mathcal{D}_{R}^{e} \neq C^{e}\left(f^{1+i_{1}+i_{2} p+\cdots+i_{e} p^{e-1}} M\right) \cdot \mathcal{D}_{R}^{e}
$$


for all $e \gg 0$. Finally, by Corollary 5.3 this is equivalent to $\frac{\left\lceil\lambda p^{e}\right\rceil-1}{p^{e}}$ being a zero of $b_{M, f}^{e}(s)$ for all $e \gg 0$.

\section{REMARK 5.5.}

(a) We recall that there are only finitely many $F$-jumping numbers in $(0,1]$ and that they are all rational. In particular, the limit over the $b_{M, f}^{e}(s)$ for $e \rightarrow \infty$ is a polynomial with rational roots.

(b) The case where $M$ is locally constant (and $R$ is smooth over a perfect field), that is, when there exists a finite étale morphism $\varphi: \operatorname{Spec} S \rightarrow$ Spec $R$ such that $\varphi^{*} M \cong \omega_{S}^{n}$ can also be directly deduced from the constant case treated in [23]. This essentially boils down to the fact that differential operators along étale morphisms are well behaved (one obtains an inclusion $\mathcal{D}_{R}^{e} \rightarrow \mathcal{D}_{S}^{e}$ and the natural map $M \rightarrow \varphi^{!} M$ is $\mathcal{D}_{R}^{e}$ linear - see [22, Theorem 2.2.5, Corollary 2.2.6] for the first statement. The latter may be extracted from [22, Theorem 2.2.10] and [3, 2.1.3]). Then one uses [25, Theorem 8.5] to see that the $F$-jumping numbers of $M$ are the same as that of $\omega_{S}$. In fact, writing this up precisely was the original motivation for this paper.

(c) Note that Mustaţă's result [23, Theorem 6.7] is valid for any $e \geqslant 1$ while we only obtain a result for $e \gg 0$.

\section{§6. A comparison with Stadnik's $b$-functions}

The goal of this section is to point out the relation of our Bernstein-Sato polynomials to the $b$-functions of Stadnik defined in [26, Definition 4.4]. Stadnik works in the context of unit $R[F]$-modules which were introduced by Lyubeznik [20] and Emerton-Kisin [14]. We briefly recall the relevant notions. A unit $R[F]$-module is an $R$-module $\mathcal{M}$ equipped with a structural isomorphism $\theta: F^{*} \mathcal{M} \cong \mathcal{M}$. A root of $\mathcal{M}$ is an $R$-module $M$ together with an injective $R$-linear map $\Phi: M \rightarrow F^{*} M$ such that $\operatorname{colim}_{e} F^{e *} M$ and $\mathcal{M}$ are isomorphic as unit $R[F]$-modules. In particular, if $M$ is a root for $\mathcal{M}$ then $\gamma_{*} M \otimes \omega_{R[t] / R}^{-1}$ is a root for $\gamma_{+} \mathcal{M}$, where $\gamma:$ Spec $R \rightarrow \operatorname{Spec} R[t]$ is the graph embedding for some hypersurface $f$ (Proposition 3.5 above shows that $\gamma_{+}$is the $\mathcal{D}$-module pushforward and $[14,14.3 .10,15.2]$ shows that the $\mathcal{D}$-module pushforward coincides with the pushforward on unit $R[F]$ modules). By abuse of notation we denote the image of the natural map $\gamma_{*} M \otimes \omega_{R[t] / R}^{-1} \rightarrow F^{e^{*}}\left(\gamma_{*} M \otimes \omega_{R[t] / R}^{-1}\right)$ again by $\gamma_{*} M \otimes \omega_{R[t] / R}^{-1}$. 
Given a sequence $i_{l}$ of integers in $\{0, \ldots, p-1\}$ we refer to $\sum_{l=0}^{e-1} i_{l} p^{l}$ as the base-p expansion of $\left(i_{1}, \ldots, i_{e}\right)$. Varying $e$ we call the number $\lim _{e \rightarrow \infty} \sum_{l=0}^{e-1} i_{l} p^{l} / p^{e}$ the $p$-weighted limit of the base-p expansion if it exists.

With this notation Stadnik defines a b-function (for the pair $\left(\gamma_{*} M \otimes\right.$ $\left.\left.\omega_{R[t] / R}^{-1}, \gamma_{+} \mathcal{M}\right)\right)$ as any polynomial $b(s) \in \mathbb{C}[s]$ with roots in $(0,1]$ that satisfies the following property:

If $\lambda$ is a root of $b(1-s)$ then there exists an integer $n$ such that for all $e \geqslant 0$ the set

$$
\left\{\left\lceil\lambda p^{e}\right\rceil-a \mid 0 \leqslant a \leqslant p^{n}\right\}
$$

contains the base $p$-expansions of the eigenvalues of the $\theta_{p^{l}}, l=0, \ldots, e-1$, on the quotient

$$
D_{R}^{e}\left[\theta_{1}, \ldots, \theta_{p^{e-1}}\right]\left(\gamma_{*} M \otimes \omega_{R[t] / R}^{-1}\right) / D_{R}^{e}\left[\theta_{1}, \ldots, \theta_{p^{e-1}}\right] t\left(\gamma_{*} M \otimes \omega_{R[t] / R}^{-1}\right) .
$$

The set of $b$-functions forms an ideal in $\mathbb{C}[s]$ and we denote its monic generator by $\tilde{b}_{M, f}(s)$.

The main result of [26] is that $\tilde{b}_{M, f}(s)$ is a nonzero polynomial with rational roots. We reprove this here using Theorem 5.4 under the additional assumption that $M \otimes \omega_{R}$ is $F$-regular and that $f$ is not a zero divisor on $M$.

REMARK 6.1. Note that $\Omega_{R[t] / R}$ is free of rank 1 and $d t=d(t-f)$. By definition $\partial_{t}$ is the differential operator in $\operatorname{Hom}_{R}\left(\Omega_{R[t] / R}, R\right)=R \oplus$ $\operatorname{Der}_{R[t] / R}$ given by the dual of $d t$. In particular, we have $\partial_{t}=\partial_{t-f}$. Hence, applying the automorphism $t \mapsto t+f$ we are precisely in the setting where our hypersurface equation is given by $t=0$ and the Euler operators are given by $\theta_{e}=t^{p^{e}} \partial_{t}^{\left[p^{e}\right]}$, where we use the inclusion $\mathcal{D}_{R[t] / R} \subseteq \mathcal{D}_{R[t]}$. The latter is the setting in which Stadnik works.

Also note that Stadnik considers the quotient

$$
D_{R}^{e}\left[t, \theta_{1}, \ldots, \theta_{p^{e-1}}\right]\left(\gamma_{*} M \otimes \omega_{R[t] / R}^{-1}\right) / D_{R}^{e}\left[t, \theta_{1}, \ldots, \theta_{p^{e-1}}\right] t\left(\gamma_{*} M \otimes \omega_{R[t] / R}^{-1}\right)
$$

but since $\left[t, \theta_{i}\right]=\theta_{i-1}$ and $t\left(\gamma_{*} M \otimes \omega_{R[t] / R}^{-1}\right)=\gamma_{*} f M \otimes \omega_{R[t] / R}^{-1}$ this quotient coincides with the one we consider.

The equivalence of our notion and that of Stadnik is obtained from the equivalence of left and right $\mathcal{D}_{S^{-}}^{e}$-modules which we recall in a special setting. For an $S$-module $M$ the $e$ th Frobenius pull back ${ }^{5} F^{e *} M=F_{*}^{e} R \otimes_{R} M$ is a

\footnotetext{
${ }^{5}$ Note that again we view $F_{*}^{e} S$ as an $S$-bimodule where the structure on the left is obtained by the ring isomorphism $S \rightarrow F_{*}^{e} S$.
} 
left $\mathcal{D}_{S}^{e}$-module via the action of $\mathcal{D}_{S}^{e}$ on $F_{*}^{e} S$. Tensoring with $\omega_{S}$ one obtains an isomorphism $\omega_{S} \otimes F^{{ }^{*}} M \cong F^{e !}\left(\omega_{S} \otimes M\right)=\operatorname{Hom}_{S}\left(F_{*}^{e} S, \omega_{S} \otimes M\right)$ and the latter naturally carries the structure of a right $\mathcal{D}_{S}^{e}$-module via the action on $F_{*}^{e} S$. Since $\omega_{S}$ is invertible this induces an equivalence of categories between left and right $\mathcal{D}_{S}^{e}$-modules.

Given a smooth ring $S$ over a perfect field $k$ we can choose a set of local coordinates $x_{1}, \ldots, x_{n}$. For $i \in \mathbb{N}^{n}$ write $\partial_{i}^{\left[m_{i}\right]}=\partial_{x_{i_{1}}}^{\left[m_{1}\right]} \cdots \partial_{x_{i_{n}}}^{\left[m_{n}\right]}$. Now given any differential operator $P$ we can write it locally as

$$
\sum_{i \in \mathbb{N}^{n}} s_{i} \partial_{i}^{\left[m_{i}\right]},
$$

where almost all $i$ are zero. Then we denote the adjoint operator

$$
\sum_{i}(-1)^{\sum_{j=1}^{n} i_{j}} \partial_{i}^{\left[m_{i}\right]} s_{i}
$$

by $P^{t}$. Finally, note that one has $(P Q)^{t}=Q^{t} P^{t}$.

Proposition 6.2. Let $S=R[t]$ for $R$ smooth over a perfect field $k$ and $M$ an $S$-module. Then for a set of local coordinates $t, x_{1}, \ldots, x_{n}$ the right $\mathcal{D}_{S}^{e}$-module structure on $\omega_{S} \otimes F^{{ }^{*}} M$ is locally given by

$$
\left(d t \wedge d x_{1} \wedge \cdots \wedge d x_{n} \otimes m\right) \cdot P=d t \wedge d x_{1} \wedge \cdots \wedge d x_{n} \otimes P^{t} m
$$

and the isomorphism $\omega_{S} \otimes F^{e *} M \rightarrow F^{e !}\left(\omega_{S} \otimes M\right)$ is $\mathcal{D}_{S}^{e}$-linear. In particular, for $1 \otimes v \otimes m \in \omega_{S / R} \otimes_{R} \omega_{R} \otimes F^{e^{*}} M$ one has $(1 \otimes v \otimes m) \cdot \theta_{p^{l}}=$ $\left(1 \otimes v \otimes-\vartheta_{p^{l}} m\right)$ for any $0 \leqslant l \leqslant e-1$.

Proof. First of all, we reduce to the case $M=S$. The $\mathcal{D}_{S}^{e}$-module structure on $F^{e *} M=F_{*}^{e} S \otimes_{S} M$ and on $F^{e !}\left(\omega_{S} \otimes M\right)=\operatorname{Hom}_{S}\left(F_{*}^{e} S, \omega_{S} \otimes\right.$ $M)$ is given by the action of $\mathcal{D}_{S}^{e}$ on $F_{*}^{e} S$. Moreover, the isomorphism $\omega_{S} \otimes F^{e^{*}} M \rightarrow F^{e^{!}}\left(\omega_{S} \otimes M\right)$ factors as the composition of the canonical isomorphisms

$$
\omega_{S} \otimes F^{e *} M \longrightarrow \omega_{S} \otimes F^{e *} S \otimes M \stackrel{\Sigma \otimes \mathrm{id}}{\longrightarrow} F^{e !} \omega_{S} \otimes M \longrightarrow F^{e !}\left(\omega_{S} \otimes M\right),
$$

where $\Sigma: \omega_{S} \otimes F^{e *} S \rightarrow F^{e !} \omega_{S}$ denotes the isomorphism $d s \otimes f \mapsto C^{e}(d s)$. $f=\left[x \mapsto \kappa^{e}(x f d s)\right]$.

Now the claim follows from [3, Proposition 1.1.7(i), Corollary 1.2.6] with $\mathcal{M}=\omega_{S}$ and $\mathcal{E}=F_{*}^{e} S$ : Since $k$ is perfect we have $\mathcal{D}_{S / k}^{e}=\mathcal{D}_{S}^{e}$. By [2, Proposition 2.2.7] the image of $\mathcal{D}_{S}^{(e)}$ in $\mathcal{D}_{S}$ corresponds to $\mathcal{D}_{S}^{e}$ so that Berthelot's results also apply to $\mathcal{D}_{S}^{e}$-modules. 
COROllary 6.3. Let $S=R[t]$ for a regular ring $R$ essentially of finite type over an $F$-finite field and $M$ an $S$-module. Then $F^{{ }^{*}} M$ admits a nontrivial $\left(-i_{1}, \ldots,-i_{e}\right)$-eigenspace for the $-\vartheta$ if and only if $F^{e !}\left(M \otimes \omega_{S}\right)$ admits a nontrivial $\left(i_{1}, \ldots, i_{e}\right)$-eigenspace for the $\theta$.

Proof. The "only if"-part is immediate from Proposition 6.2. Conversely, $F^{e !}\left(M \otimes \omega_{S}\right) \otimes \omega_{S}^{-1}$ is canonically isomorphic to $F^{{ }^{*}} M$ and a similar argument applies in this case.

Lemma 6.4. Let $(M, \Phi)$ be a root of a unit $R[F]$-module $\mathcal{M}$ and $\gamma$ : Spec $R \rightarrow \operatorname{Spec} S=R[t]$ a closed immersion and $\left(\gamma_{*} M \otimes \omega_{S / R}^{-1}, \Phi \otimes \mathrm{id}\right)$ the corresponding root for $\gamma_{+} \mathcal{M}$.

Then $\left(\gamma_{*}\left(\omega_{R} \otimes M\right), \tilde{C}\right)$ and $\left(\omega_{R} \otimes M, C\right)$ are naturally Cartier modules and the map $\tilde{C}: \gamma_{*}\left(\omega_{R} \otimes M\right) \rightarrow F^{!} \gamma_{*} \omega_{R} \otimes M$ is given by the composition of $\gamma_{*}\left(\omega_{R} \otimes M\right) \rightarrow \gamma_{*} F^{!}\left(\omega_{R} \otimes M\right) \rightarrow F^{!}\left(\gamma_{*} \omega_{R} \otimes M\right)$, where the first map is $\gamma_{*} C$ and the second is the composition of maps described in Proposition 3.3. In particular, if $N$ denotes the image of the natural map $\gamma_{*} M \otimes \omega_{R[t] / R}^{-1} \rightarrow$ $F^{e^{*}}\left(\gamma_{*} M \otimes \omega_{R[t] / R}^{-1}\right)$ then $\omega_{R[t]} \otimes N$ is the image of the natural map $\gamma_{*}\left(\omega_{R} \otimes\right.$ $M) \rightarrow F^{e !}\left(\gamma_{*} \omega_{R} \otimes M\right)$.

Proof. We shorten $\omega_{R} \otimes M$ to $M^{\prime}$. It is easy to see that the Cartier structure given on $\gamma_{*} M^{\prime}$ is the one induced from $M^{\prime}$ by

$$
F_{*} \gamma_{*} M^{\prime} \stackrel{\sim}{\longrightarrow} \gamma_{*} F_{*} M^{\prime} \stackrel{\gamma_{*} \kappa_{M^{\prime}}}{\longrightarrow} \gamma_{*} M^{\prime} .
$$

Hence, one may reduce the problem to checking that given a Cartier module $(A, \kappa)$ with adjoint $C$ the adjoint of the structural map of $\gamma_{*} A$ is given by the composition of the map described in Proposition 3.3 with $\gamma_{*} C$. This is an easy computation which will be left to the reader.

LEMMA 6.5. Let $R$ be smooth over a perfect field and $f \in R$ a hypersurface. Let $\gamma: \operatorname{Spec} R \rightarrow \operatorname{Spec} R[t]$ be the graph embedding along $f$. Given an $R$-module $M$ we have

$$
\begin{aligned}
\omega_{R[t]} & \otimes\left(\mathcal{D}_{R}^{e}\left[\theta_{1}, \theta_{p}, \ldots, \theta_{p^{e-1}}\right] \gamma_{*} M \otimes \omega_{R[t] / R}^{-1}\right) \\
& =\left(\gamma_{*} \omega_{R} \otimes M\right) \mathcal{D}_{R}^{e}\left[\theta_{1}, \theta_{p}, \ldots, \theta_{p^{e-1}}\right] .
\end{aligned}
$$

Proof. According to our established abuse of notation we have to show that for $m$ in the image of $\gamma_{*} M \otimes \omega_{R[t] / R}^{-1} \rightarrow F^{e *} \gamma_{*} M \otimes \omega_{R[t] / R}^{-1}$ one has that $\omega \otimes P \cdot m$ for any $P \in D_{R}^{e}\left[\theta_{1}, \ldots, \theta_{p^{e-1}}\right]$ is contained in the $\mathcal{D}_{R}^{e}\left[\theta_{1}, \ldots, \theta_{p^{e-1}}\right]$-module generated by the image of $\gamma_{*}\left(\omega_{R} \otimes M\right) \rightarrow$ $F^{e !} \gamma_{*}\left(\omega_{R} \otimes M\right)$ and vice versa. We may verify this locally and then it follows 
from Proposition 6.2 and the fact that $\mathcal{D}_{R}^{e}\left[\theta_{1}, \ldots, \theta_{p^{e-1}}\right]$ is closed under taking adjoints. This is clear for $\mathcal{D}_{R}^{e}$ and for $\theta_{p^{i}}$ one has for the transposed operator $\left(\theta_{p^{i}}\right)^{t}=-\vartheta_{p^{i}}=-\left(1+\theta_{p^{i}}\right)$ by Lemma 1.1 (i).

COROLlary 6.6. In the situation of Lemma 6.5 we have an isomorphism

$$
\begin{gathered}
\left(\mathcal{D}_{R}^{e}\left[\theta_{1}, \ldots, \theta_{p^{e-1}}\right] \gamma_{*} M \otimes \omega_{R[t] / R}^{-1} / \mathcal{D}_{R}^{e}\left[\theta_{1}, \ldots, \theta_{p^{e-1}}\right] t \gamma_{*} M \otimes \omega_{R[t] / R}^{-1}\right) \otimes \omega_{R[t]} \\
\cong\left(\gamma_{*} \omega_{R} \otimes M\right) \mathcal{D}_{R}^{e}\left[\theta_{1}, \ldots, \theta_{p^{e-1}}\right] /\left(\gamma_{*} \omega_{R} \otimes M\right) t \mathcal{D}_{R}^{e}\left[\theta_{1}, \ldots, \theta_{p^{e-1}}\right]
\end{gathered}
$$

Proof. Note that

$$
\left(t \gamma_{*} M\right) \mathcal{D}_{R}^{e}\left[\theta_{1}, \ldots, \theta_{p^{e-1}}\right]=\gamma_{*}\left(f M \mathcal{D}_{R}^{e}\right) \mathcal{D}_{R}^{e}\left[\theta_{1}, \ldots, \theta_{p^{e-1}}\right]
$$

so that the claim follows from Lemma 6.5 and tensoring the obvious short exact sequence with $\omega_{S}$.

Corollary 6.7. The $\mathcal{D}_{R}^{e}\left[\theta_{1}, \ldots, \theta_{p^{e-1}}\right]$-module

$$
D_{R}^{e}\left[\theta_{1}, \ldots, \theta_{p^{e-1}}\right] \gamma_{*} M \otimes \omega_{R[t] / R}^{-1} / D_{R}^{e}\left[\theta_{1}, \ldots, \theta_{p^{e-1}}\right] t \gamma_{*} M \otimes \omega_{R[t] / R}^{-1}
$$

has a nontrivial $\vartheta$-eigenspace with eigenvalue $\left(-i_{1}, \ldots,-i_{e}\right)$ if and only if the right $\mathcal{D}_{R}^{e}\left[\theta_{1}, \ldots, \theta_{p^{e-1}}\right]$-module

$$
\left(\gamma_{*} \omega_{R} \otimes M\right) D_{R}^{e}\left[\theta_{1}, \ldots, \theta_{p^{e-1}}\right] /\left(\gamma_{*} \omega_{R} \otimes M\right) t D_{R}^{e}\left[\theta_{1}, \ldots, \theta_{p^{e-1}}\right]
$$

has a nontrivial $\theta$-eigenspace with eigenvalue $\left(i_{1}, \ldots, i_{e}\right)$.

Proof. Note that the left module embeds into $\mathcal{D}_{R}^{e} \gamma_{*} M \otimes \omega_{R[t] / R}^{-1} / \gamma_{*} M \otimes$ $\omega_{R[t] / R}^{-1}$ and similarly for the right module. Then the claim follows from Corollaries 6.3 and 6.6

REMARK 6.8. One should be able to obtain a similar correspondence between right and left $\mathcal{D}_{R}^{e}\left[\theta_{1}, \ldots, \theta_{p^{e-1}}\right]$-modules under the weaker assumption that $R$ is regular, essentially of finite type over an $F$-finite field. However, in this case one cannot appeal to Berthelot's results. Since Stadnik works under the assumptions that $R$ is smooth over a perfect field we did not pursue this further.

We now have the necessary ingredients to state and prove the main results of this section.

TheOREM 6.9. Let $\gamma:$ Spec $R \rightarrow$ Spec $R[t]$ be the graph embedding along a hypersurface $f$ and let $(M, \Phi)$ be a root of a unit $R[F]$-module $\mathcal{M}$. Assume 
that the Cartier module $M \otimes \omega_{R}$ is $F$-regular ${ }^{6}$ and that $f$ is not a zero divisor on $M$. Then if $\mu_{e}$ denotes the base-p expansions of the eigenvalues of the $\theta_{p^{l}}$ operating on the left modules

$$
\mathcal{D}_{R}^{e}\left[\theta_{1}, \ldots, \theta_{p^{e-1}}\right] \gamma_{*} M \otimes \omega_{R[t] / R}^{-1} / \mathcal{D}_{R}^{e}\left[\theta_{1}, \ldots, \theta_{p^{e-1}}\right] \gamma_{*} t M \otimes \omega_{R[t] / R}^{-1}
$$

and $\lambda_{e}$ denotes the base-p expansions of the eigenvalues of $\theta_{p^{l}}$ operating on the right modules

$$
\left(\gamma_{*} \omega_{R} \otimes M\right) \mathcal{D}_{R}^{e}\left[\theta_{1}, \ldots, \theta_{p^{e-1}}\right] /\left(\gamma_{*} \omega_{R} \otimes M\right) t \mathcal{D}_{R}^{e}\left[\theta_{1}, \ldots, \theta_{p^{e-1}}\right]
$$

one has the following relation $\mu_{e}+\lambda_{e}=p^{e}-1$ and

$$
\lim _{e \rightarrow \infty} \frac{\mu_{e}}{p^{e}}=1-\lim _{e \rightarrow \infty} \frac{\lambda_{e}}{p^{e}} .
$$

In particular, the $p$-weighted limit of the $\mu_{e}$ exists.

Proof. If $\theta_{p^{l}}$ operates from the left with eigenvalue $-i_{l}-1$ then by Corollary 6.7 and the relation $\theta_{p^{l}}+1=\vartheta_{p^{l}}$ we have that $\theta_{p^{l}}$ operates via $i_{l}$ on the right. So we get $\mu_{e}=\sum_{l=0}^{e-1}\left(p-1-i_{l}\right) p^{l}$ as the base- $p$ expansion for the operation of the $\theta_{p^{l}}$ on the left. Similarly, we have $\lambda_{e}=\sum_{l=0}^{e-1} i_{l} p^{l}$.

By Theorem 5.4 the $p$-weighted limit over the $\lambda_{e}$ exists. Moreover,

$$
\lim _{e \rightarrow \infty} \sum_{l=0}^{e-1} \frac{(p-1) p^{l}}{p^{e}}=1
$$

so that the claim follows.

We denote the limit for $e \rightarrow \infty$ of the polynomials $b_{M, f}^{e}(s)$ introduced in Definition 3.7 by $b_{M, f}(s)$. With this notation we can now compare Stadnik's notion of Bernstein-Sato polynomial to our notion:

Corollary 6.10. Assume the situation of Theorem 6.9. Let $\lambda_{1}, \ldots, \lambda_{m}$ be p-weighted limits of the base-p expansions of the eigenvalues of the $\theta_{p^{l}}$ acting on

$$
\left(\gamma_{*} \omega_{R} \otimes M\right) D_{R}^{e}\left[\theta_{1}, \ldots, \theta_{p^{e-1}}\right] /\left(\gamma_{*} \omega_{R} \otimes M\right) t D_{R}^{e}\left[\theta_{1}, \ldots, \theta_{p^{e-1}}\right] .
$$

Then $\tilde{b}_{M, f}(s)=\prod_{i}\left(s-\lambda_{i}\right)$. In particular, $\tilde{b}_{M, f}(s)=b_{M, f}(s)$

\footnotetext{
${ }^{6}$ If $M$ denotes the unique minimal root of $\mathcal{M}$ in the sense of [5, Definition 2.7] then this just means that $M$ is generically simple. That is, any submodule $N$ of $M$ for which $N \rightarrow F^{*} M$ factors through $N \rightarrow F^{*} N$ which agrees at all generic points of $\operatorname{Supp} M$ with $M$ coincides with $M$.
} 
Proof. First of all, we use Theorem 6.9 to ensure that the $p$-weighted limits actually do exist. Recall from the discussion before Theorem 5.4 that for all $e \geqslant 1$ the base- $p$ expansions of the eigenvalues of $\theta$ are given by $\left\lceil\lambda p^{e}\right\rceil-1$. This implies that $b(s)$ is a Bernstein-Sato polynomial.

In the other direction we have to show that $b(s)$ is minimal in the sense that we may not omit any of the $\lambda_{i}$. Assume that we have omitted $\lambda_{m}$ and for some $n \geqslant 0$ the set $\left\{\left\lceil\lambda_{m} p^{e}\right\rceil-a \mid 0 \leqslant a \leqslant p^{n}\right\}$ is contained in the set $\left\{\left\lceil\lambda_{i} p^{e}\right\rceil-a \mid 0 \leqslant a \leqslant p^{n}, 1 \leqslant i \leqslant m-1\right\}$. In particular, the $\left\lceil\lambda_{m} p^{e}\right\rceil-1$ are all contained in this set. Since all parameters except $e$ of this set are finite we may assume that $\left\lceil\lambda_{m} p^{e}\right\rceil-1=\left\lceil\lambda_{i} p^{e}\right\rceil-a$ for some fixed $a, i$ and infinitely many $e$. Dividing by $p^{e}$ and passing to the limit $e \rightarrow \infty$ yields $\lambda_{i}=\lambda_{m}-$ a contradiction.

Acknowledgments. The idea for this paper was conceived while Axel Stäbler was a guest of the University of Michigan. In particular, he would like to thank Mircea Mustaţă and Karen Smith for making this visit possible.

\section{REFERENCES}

[1] J. Alvarez-Montaner, M. Blickle and G. Lyubeznik, Generators of D-modules in positive characteristic, Math. Res. Lett. 12(4) (2005), 459-473.

[2] P. Berthelot, $\mathcal{D}$-module arithmétiques I: Opérateurs différentiels de nivau fini, Ann. Sci. Éc. Norm. Supér. 29(2) (1996), 185-272.

[3] P. Berthelot, D-module arithmétiques II: Descente par Frobenius, Mém. Soc. Math. Fr. (N.S.) 81 (2000), 1-136.

[4] M. Blickle, The intersection homology D-module in finite characteristic, Ph.D. thesis, University of Michigan, 2001.

[5] M. Blickle, Minimal $\gamma$-sheaves, Algebra Number Theory 2(3) (2008), 347-368.

[6] M. Blickle, Test ideals via $p^{-e}$-linear maps, J. Algebraic Geom. 22(1) (2013), 49-83.

[7] M. Blickle and G. Böckle, Cartier modules: Finiteness results, J. Reine Angew. Math. 661 (2011), 85-123.

[8] M. Blickle and G. Böckle, A corresondence between crystals over function fields and Emerton-Kisin's unit $\mathcal{O}_{X}[F]$-modules, preprint, 2012.

[9] M. Blickle, M. Mustaţă and K. Smith, Discreteness and rationality of F-thresholds, Michigan Math. J. 57 (2008), 43-61.

[10] M. Blickle, M. Mustaţă and K. Smith, F-thresholds of hypersurfaces, Trans. Amer. Math. Soc. 361(12) (2009), 6549-6565.

[11] N. Budur, "On the V-filtration of D-modules", in Geometric methods in algebra and number theory, Progress in Mathematics 235 (eds. F. Bogomolov and Yu. Tschinkel) Birkhäuser, Boston, 2005.

[12] N. Budur and M. Saito, Multiplier ideals, V-filtration and spectrum, J. Algebraic Geom. 14(2) (2005), 269-282.

[13] S. U. Chase, On the homological dimension of algebras of differential operators, Comm. Algebra 1(5) (1974), 351-363.

[14] M. Emerton and M. Kisin, The Riemann-Hilbert correspondence for unit F-crystals, Asterisque 293 (2004). 
[15] A. Grothendieck and J. Dieudonné, Eléments de géométrie algébrique IV, Quatrième partie, Publ. Math. Inst. Hautes Études Sci. 32 (1967), 361 pp.

[16] M. Kashiwara, Vanishing cycle sheaves and holonomic systems of differential equations, Lecture Notes in Mathematics 1016, Springer, 1983.

[17] E. Kunz, Characterizations of regular local rings of characteristic p, Amer. J. Math. 91 (1969), 772-784.

[18] T. Y. Lam, Lecture on Modules and Rings, Springer, New York, 1999.

[19] E. Lucas, Théorie des Fonctions Numériques Simplement Périodiques, Amer. J. Math. 1(2) (1878), 197-240.

[20] G. Lyubeznik, F-modules: applications to local cohomology and D-modules in characteristic $p>0$, J. Reine Angew. Math. 491 (1997), 65-130.

[21] B. Malgrange, Polynôme de Bernstein-Sato et cohomologie évanescente, Astérisque 101-102(2-3) (1983), 243-267.

[22] G. Másson, Rings of differential operators and étale homomorphisms, Ph.D. thesis, Massachusetts Institue of Technology, 1991.

[23] M. Mustaţă, Bernstein-Sato polynomials in positive characteristic, J. Algebra 321(1) (2009), 128-151.

[24] C. Sabbah, "D-modules et cycles évanescents (d'après B. Malgrange et M. Kashiwara)", in Géométrie algébrique et applications III, Travaux en cours 24, 1987, 53-98.

[25] A. Stäbler, V-filtrations in positive characteristic and test modules, Trans. Amer. Math. Soc., electronically published on January 27, 2016, doi:10.1090/tran/6632 (to appear in print).

[26] Th. Stadnik, The lemma on b-functions in positive characteristic, preprint, 2012, arXiv: 1206.4039 .

[27] T. Torrelli, Intersection homology D-module and Bernstein polynomials associated with a complete intersection, Publ. Res. Inst. Math. Sci. 45(2) (2009), 645-660.

Manuel Blickle

Johannes Gutenberg-Universität Mainz

Fachbereich 08

Staudingerweg 9

55099 Mainz

Germany

blicklem@uni-mainz.de

Axel Stäbler

Johannes Gutenberg-Universität Mainz

Fachbereich 08

Staudingerweg 9

55099 Mainz

Germany

staebler@uni-mainz.de 